\title{
THE PERMANENT FUNCTION AS AN INNER PRODUCT ${ }^{1}$
}

BY MARVIN MARCUS AND MORRIS NEWMAN

Communicated by A. M. Gleason, November 25, 1960

Let $U$ be a unitary $n$-space with inner product $(x, y)$ and let $U^{(k)}$ denote the space of totally symmetric multilinear functionals of $k$-tuples of vectors from $U$. If $x_{1}, \cdots, x_{k}$ are vectors in $U$, then the element $\phi=x_{1} \otimes \cdots \otimes x_{k} \in U^{(k)}$ is defined by

$$
\phi\left(u_{1}, \cdots, u_{k}\right)=\sum_{\sigma} \prod_{i=1}^{k}\left(u_{i}, x_{\sigma(i)}\right)
$$

where the summation extends over all $k$ ! permutations $\sigma$ of $1, \cdots, k$. The conjugate bilinear functional

(1) $\left(x_{1} \otimes \cdots \otimes x_{k}, y_{1} \otimes \cdots \otimes y_{k}\right)=\operatorname{per}\left[\left(x_{i}, y_{j}\right)\right] \quad i, j=1, \cdots, k$ is an inner product in $U^{(k)}$. Here per $(A)$ denotes the permanent of $A$,

$$
\operatorname{per}(A)=\sum_{\sigma} \prod_{i=1}^{k} a_{i \sigma(i)}
$$

By applying the Schwarz inequality to the inner product (1) and making a careful investigation of the case of equality we obtain the

THEOREM. If $x_{1}, \cdots, x_{k}$ and $y_{1}, \cdots, y_{k}$ are two sets of $k$ vectors each, then

$$
\left|\operatorname{per}\left[\left(x_{i}, y_{j}\right)\right]\right|^{2} \leqq \operatorname{per}\left[\left(x_{i}, x_{j}\right)\right] \operatorname{per}\left[\left(y_{i}, y_{j}\right)\right] .
$$

If the zero vector occurs in neither set then equality in (2) implies that the space spanned by both sets is the same. If the sets are linearly independent then equality in (2) implies that except for order and scalar factors the sets are termwise identical.

A number of corollaries follow easily. The first of these is a partial answer to a conjecture of van der Waerden [1].

I. If $A$ is an n-square doubly stochastic positive semi-definite symmetric matrix then

$$
\operatorname{per}(A) \geqq n ! / n^{n}
$$

with equality if and only if $A$ is the matrix all of whose entries are $1 / n$.

II. If $A$ and $B$ are $n$-square complex matrices then

1 This work was supported in part by the Office of Naval Research. 


$$
|\operatorname{per}(A B)|^{2} \leqq \operatorname{per}\left(A A^{*}\right) \operatorname{per}\left(B^{*} B\right) \text {. }
$$

If $A$ and $B$ are nonsingular then equality can hold if and only if $B^{*}=D P A$ where $D$ is a diagonal matrix and $P$ is a permutation matrix.

III. If $A$ is an $n$-square positive semi-definite hermitian matrix then

$$
\operatorname{per}(A) \geqq \operatorname{det} A
$$

with equality if and only if $A$ has a zero row or $A$ is a diagonal matrix. Thus $\sum^{\prime} \prod a_{i \sigma(i)} \geqq 0$ where $\sum^{\prime}$ denotes the sum over all odd permutations $\sigma$. Equality holds if and only if $A$ has a zero row or $A$ is diagonal.

IV. If $U$ is unitary then

$$
|\operatorname{per}(U)| \leqq 1
$$

with equality if and only if $U$ is a generalized permutation matrix.

Some further applications are made, e.g., to obtain bounds for the number of distinct representatives of a $(v, k, \lambda)$ configuration.

\section{REFERENCE}

1. Marvin Marcus and Morris Newman, Permanents of doubly stochastic matrices, Proceedings of Symposia in Applied Mathematics, vol. 10, American Mathematical Society, 1960, pp. 169-174.

National Bureau of Standards and

The University of British Columbia 